\title{
Nutritional psychiatry: The next frontier in mental health treatment
}

\author{
Robin Goh ${ }^{1}$ MMed (Psych), Shang Cong Lim ${ }^{1}$ MRCPsych
}

The World Health Organization has envisioned for every human being's fundamental right to be able to enjoy the highest attainable standard of health, even in the presence of disease or infirmary. Singaporeans' average life expectancy is projected to reach 85.4 years by the year $2040^{1}$ and the burden of disease will inevitably rise. The second Singapore Mental Health Study conducted in 2016 revealed 1 in 7 Singaporeans (13.9\%) had experienced a mental disorder in their lifetime, ${ }^{2}$ and this was an increase from the 2010 study where it was reported at 1 in 8 Singaporeans (12\%). Of interest, more than threequarters of them did not seek professional help. There was also considerable treatment delay among those who sought help. Nutritional psychiatry is a developing field that explores how dietary intake patterns affect mood, behaviour and mental health. This novel approach in psychiatric management may have a role in bridging the treatment gap through the prevention of mental disorders, as part of an integrated treatment algorithm, or even as an alternative treatment modality.

Pharmacological interventions and psychotherapies have remained the main treatment modalities for psychiatric patients seeking help. However, most individuals who are suffering from mental disorders may not be aware of their conditions and the resources available. ${ }^{3}$ They may also be in denial or fear stigmatisation following psychiatric consultations. There is increasing evidence that indicates a strong association between a poor diet and the exacerbation of mood disorders such as anxiety and depression. However, establishing a direct connection is difficult due to the multifactorial nature of having mental health conditions.

The Singapore Chinese Health Study, ${ }^{4}$ which was one of the largest population-based cohorts in Southeast Asia, interviewed over 60,000 individuals in the 1990s about their life and food habits. During the 17 years of follow-up, researchers collected the participants' dietary information based on the Dietary Approaches to Stop Hypertension (DASH) diet and found that individuals with a diet resembling the DASH dietary pattern were at 31-26\% lower risk of death due to heart attack and stroke, even after controlling for many other coexisting characteristics of participants at baseline. This study demonstrated the close relationship between diet and diseases. The DASH diet was originally developed to prevent hypertension, but it had been extrapolated to investigate relationships with mental health disorders ${ }^{5}$ on the basis that hypertension and some mental disorders do share common modifiable risk factors and underlying biological mechanisms.

Using food as an alternative for the prevention or treatment of mental disorders is not a foreign concept. Hypericum perforatum, commonly known as perforate St John's wort, has been used in herbalism for centuries. The plant contains hyperforin, which has been shown to inhibit the neuronal uptake of serotonin, norepinephrine and dopamine. A 2015 meta-analysis review ${ }^{6}$ also concluded perforate St John's wort to be superior to placebo in treating depression and having fewer adverse effects than other antidepressants. It is approved as a dietary supplement by the Food and Drug Administration (FDA) in the US for the treatment of depression.

Nutritional psychiatry provides new insights into the treatment of mental disorders and is aligned with the principles of integrative health, which is to treat the patient as a whole person and not just the constellation of symptoms. Diets consisting of unprocessed food (lower DASH score) do contain more essential vitamins, minerals and nutrients that significantly support the body's biological and physiological processes. They are less inflammatory to the gut environment (microbiome) and support the sustainment of its probiotic population.

Probiotics in the gut have been hypothesised to exert their therapeutic effects on the central nervous system by improving the integrity of the gastrointestinal lining, reducing the ability of endotoxins to leak into the bloodstream and in turn, decreasing global inflammation. ${ }^{7}$ This reduction may result in the improved regulation of the hypothalamic-pituitary-adrenal axis and neurotransmitter activity. Serotonin, which is bio-

\footnotetext{
${ }^{1}$ Institute of Mental Health, Singapore

Correspondence: Dr Goh Kye Hock Robin, Department of Developmental Psychiatry, Institute of Mental Health, Buangkok Green Medical Park, 10 Buangkok View, Singapore 539747.

Email: robin_kh_goh@imh.com.sg
} 
synthesised both in the central nervous system and the gastrointestinal tract, is increased with healthy gut microbiome and may reduce depressive symptoms.

The SMILES (Supporting the Modification of lifestyle in Lowered Emotional States) trial ${ }^{8}$ investigated the efficacy of a dietary improvement programme for the treatment of major depressive episodes by comparing 33 depressive candidates on diet intervention against 34 controls. Both groups were allowed to continue their usual psychiatric treatments and followed up for 12 weeks. A modified Mediterranean diet (which resembled DASH diet) was administered to the treatment group; the participants were given higher portions of vegetables, fruits and other unprocessed food such as whole grains, beans and nuts, together with less refined carbohydrates and processed meats. Gluten was also excluded. At the end of the trial, the dietary support group demonstrated significantly greater improvement between the baseline and 12 weeks on the Montgomery-Asberg Depression Rating Scale compared with the control.

The road to recovery for mental health patients is often protracted. They have to reconstruct their life plans and reach a new balance. ${ }^{9}$ The study by Lee et al. alluded to the difficulties in their journey and the often ignored dietary management. ${ }^{10}$ There was a common misconception that nutritious food was bland and costly, and hence might not be easily accepted as a meal alternative. The results gave a startling realisation that females and those of an older age range of 41 to 65 years old in both depressive and psychotic disorder groups were putting more efforts in their meals. The likely postulation could be them becoming more health conscious after having severe mental disorders. The younger male demographics who did not report good dietary habits matched the profile of new onset psychotic patients. More can be done to educate them to enable a healthier relationship with their conditions.

Singapore has existing peer support ${ }^{11}$ movements for mental health patients. The peer support specialists are patients who have recovered or are stable and they have demonstrated good stewardship qualities. They can be given basic dietary training by dietitians so that they are able to advise their clients. However, existing hospital dietician referrals are limited to medical nutritional conditions such as malnourishment or obesity; dietary modifications are not listed as adjunct treatments for mental health conditions.
Nutritional psychiatry is a nascent field in psychiatric treatment and will require more research and evidence before it can be considered a mainstream option. As medical practitioners, we should be open-minded about the possibility that having a healthy diet may alleviate mental health symptoms, and advocate dietary modifications. The road to mental health recovery is often long and protracted. However, Nutritional psychiatry can now be a game changer and modify the entire mental health trajectory by being a primary prevention modality, through promoting healthier diets.

\section{REFERENCES}

1. Institute for Health Metrics and Evaluation (IHME). How healthy will we be in 2040? 16 October 2018. Available at: http:// www.healthdata.org/news-release/how-healthy-will-we-be-2040. Accessed on 26 May 2021.

2. Subramaniam M, Abdin E, Vaingankar JA, et al. Tracking the mental health of a nation: prevalence and correlates of mental disorders in the second Singapore mental health study. Epidemiol Psychiatr Sci 2019;29:e29.

3. Subramaniam M, Abdin E, Chong SA. Minding the treatment gap: results of the Singapore Mental Health Study. Soc Psychiatry Psychiatr Epidemiol 2020;55:1415-24.

4. Chen GC, Koh WP, Neelakantan N, et al. Diet Quality Indices and Risk of Type 2 Diabetes Mellitus: The Singapore Chinese Health Study. Am J Epidemiol 2018;187:2651-61.

5. Perez-Cornago A, Sanchez-Villegas A, Bes-Rastrollo M, et al. Relationship between adherence to Dietary Approaches to Stop Hypertension (DASH) diet indices and incidence of depression up to 8 years of follow-up. Public Health Nutr 2017;20:2383-92.

6. Linde K, Kriston L, Rücker G, et al. Efficacy and acceptability of pharmacological treatments for depressive disorders in primary care: systematic review and network meta-analysis. Ann Fam Med 2015;13:69-79.

7. Wallace CJK, R Milev. The effects of probiotics on depressive symptoms in humans: A systematic review. Ann Gen Psychiatry 2017;16:14.

8. Jacka FN, O'Neil A, Opie R, et al. A randomized controlled trial of dietary improvement for adults with major depression (the 'SMILES' trial). BMC Med 2017;15:23.

9. Vaingankar JA, Cetty L, Subramaniam M, et al. Recovery in Psychosis: Perspectives of Clients with First Episode Psychosis. Acad Med Singap 2020;49:186-98.

10. Lee YY, Seet V, Whitton C, et al. Dietary intake of persons with depressive and psychoticdisorders among a multiethnic Asian population. Ann Acad Med Singap 2021;50:379-89.

11. Lee YY, Ang SY, Chua HC, et al. Peer Support in Mental Health: A Growing Movement in Singapore. Ann Acad Med Singap 2019;48:95-7. 